\title{
High Spin Systems with Orbital Degeneracy
}

\author{
Shun-Qing Shen, ${ }^{1,4}$ X. C. Xie, ${ }^{2,5}$ and F.C. Zhang ${ }^{3}$ \\ ${ }^{1}$ Department of Physics, The University of Hong Kong, Pokfulam, Hong Kong \\ ${ }^{2}$ Department of Physics, Oklahoma State University, Stillwater, Oklahoma 74078 \\ ${ }^{3}$ Department of Physics, University of Cincinnati, Cincinnati, Ohio 45221 \\ ${ }^{4}$ Institute of Physics, Chinese Academy of Sciences, Beijing 100080, China \\ ${ }^{5}$ International Center for Quantum Structure, Chinese Academy of Sciences, Beijing, China
}

(Received 28 August 2001; published 26 December 2001)

\begin{abstract}
High-spin systems with orbital degeneracy are studied in the large spin limit. In the absence of Hund's coupling, the classical spin model is mapped onto disconnected orbital systems with spins up and down, respectively. The ground state of the isotropic model is an orbital valence bond state where each bond is an orbital singlet with parallel spins, and neighboring bonds interact antiferromagnetically. Possible relevance to the transition metal oxides is discussed.
\end{abstract}

DOI: 10.1103/PhysRevLett.88.027201

In many transition metal oxides, there is an orbital degeneracy in the electron occupation energy, resulting in rich and novel magnetic phenomena [1]. The orbital ordering and orbital density wave have been observed experimentally in a family of manganites [2]. In these systems, the spin coupling depends on the electron's orbital occupations. The simplest model to describe spin- $1 / 2$ systems with twofold orbital degeneracy is the SU(4) model where the Hund's rule coupling and the orbital anisotropy are neglected [3,4]. Some transition metal oxides, such as manganites and lanthanum vanadium oxides, have higher spins, which has attracted much theoretical interest [5-9]. Mathematical models for high spin systems are generally complicated. The Hund's rule coupling favors ferromagnetic (FM) spins, and the anisotropy of the electron hopping integrals breaks orbital SU(2) symmetry. While the phase diagrams of these complex spin systems are rich, there is lack of a tractable method to systematically study these systems. In this Letter, we study a class of spin systems with twofold orbital degeneracy in the limits of large spin. Our model can be derived from a lattice of ions with high spins ferromagnetically coupled to the strongly correlated electrons with double orbital degeneracy. In the classical limit of spin, such a system can be mapped onto two decoupled black and white subsystems, representing classical spin up and down, respectively. This provides an efficient method to study the ground states [10]. We apply this method to study the case where the electron hopping integrals are isotropic and use the linearized spin wave theory to examine the effects of the quantum spin fluctuation. The ground state in a 1D chain is a spin disordered valence bond state, in which each bond is an orbital valence bond (orbital singlet, parallel spins) and neighboring bonds interact antiferromagnetically. The ground states in a 2D square lattice and in a 3D cubic lattice are spin antiferromagnetically ordered orbital valence bond (OVB) states. We discuss the possible relevance of the theory to some transition metal oxides.
PACS numbers: $75.10 . \mathrm{Hk}$

We start with a Hamiltonian describing correlated electrons coupled to the spins of local ions,

$$
\begin{aligned}
H= & t \sum_{\langle i j\rangle, \alpha, \sigma}\left(c_{i \alpha \sigma}^{\dagger} c_{j \alpha \sigma}+c_{j \alpha \sigma}^{\dagger} c_{i \alpha \sigma}\right) \\
& +U \sum_{i, \alpha \sigma \neq \alpha^{\prime} \sigma^{\prime}} n_{i \alpha \sigma} n_{i \alpha^{\prime} \sigma^{\prime}}-\sum_{i, \alpha} J_{H} \mathbf{S}_{i}^{(e)} \cdot \mathbf{S}_{i}^{(\text {ion })} .
\end{aligned}
$$

In the above equation, $\alpha=1,2$ are the orbital indices and $\sigma$ is the electron spin. The sum over $\langle i j\rangle$ runs all the nearest neighbor (nn) pairs. The electronic part of the Hamiltonian is a generalized Hubbard Hamiltonian with twofold orbital degeneracy, where the Hund's rule coupling and anisotropy of the electron hopping integrals are neglected. $\mathbf{S}_{i}^{(e)}$ and $\mathbf{S}_{i}^{(\text {ion })}$ are the spin operators of the electron and the ion at site $i$, respectively. We consider $J_{H} \geq 0$, consistent with the Hund's rule. In the strong coupling limit, $U \gg t, s J_{H}$, with $s$ the quantum number of the ion spin, the projection perturbation theory may be applied to study low energy physics of the system. At the filling of one electron per site, and up to the order of $t^{2} / U$, this leads to an effective Hamiltonian [11],

$$
\begin{aligned}
H_{p}= & \frac{J}{2} \sum_{\langle i, j\rangle}\left(2 \mathbf{S}_{i}^{(e)} \cdot \mathbf{S}_{j}^{(e)}+1 / 2\right)\left(2 \mathbf{T}_{i} \cdot \mathbf{T}_{j}+1 / 2\right) \\
& -\sum_{i} J_{H} \mathbf{S}_{i}^{(e)} \cdot \mathbf{S}_{i}^{(\text {ion })}
\end{aligned}
$$

where $\mathbf{T}_{i}$ is the electron orbital operator, $J=4 t^{2} / U$. Furthermore, in the case $s J_{H}>t^{2} / U$, the electron and ion spins strongly bind ferromagnetically to form a state with total spin $s_{t}=s+1 / 2$. The effective Hamiltonian within this Hilbert space of total spin $s_{t}$ can be obtained by applying the projection operator [12],

$$
P=\prod_{i} \frac{2 \mathbf{S}_{i}^{(e)} \cdot \mathbf{S}_{i}^{(\text {ion })}+(s+1)}{2 s+1} .
$$


The new coupled spin-orbital model is found to be

$$
\begin{aligned}
H_{\text {eff }} & \equiv P H_{p} P \\
& =\frac{J}{2 s_{t}^{2}} \sum_{\langle i j\rangle}\left(\mathbf{S}_{i} \cdot \mathbf{S}_{j}+s_{t}^{2}\right)\left(\mathbf{T}_{i} \cdot \mathbf{T}_{j}+1 / 4\right)
\end{aligned}
$$

with $\mathbf{S}_{i}=\mathbf{S}_{i}^{(e)}+\mathbf{S}_{i}^{(\text {ion })}$ the total spin operator with maximal eigenvalue $s_{t}$ at site $i$.

The model has $\mathrm{SU}(2) \times \mathrm{SU}(2)$ symmetry, representing rotational invariance in both spin and orbital spaces. A special case is at $s_{t}=1 / 2$, corresponding to $\mathbf{S}_{i}^{(\text {ion })}=0$. In that case, the Hamiltonian possesses a higher SU(4) symmetry and the model has been studied extensively. In this Letter, we focus on another limit where $s_{t} \gg 1$. We start with the classical spin to replace $\mathbf{S}_{i}$ by a classical vector of length $s_{t}$ described by two angles $\theta_{i}$ and $\phi_{i}$. The Hamiltonian then reads

$$
H_{c}=J \sum_{\langle i j\rangle} \cos ^{2} \frac{\Theta_{i j}}{2}\left(\mathbf{T}_{i} \cdot \mathbf{T}_{j}+1 / 4\right),
$$

where $\Theta_{i j}$ is the angle between the two spin vectors. To investigate the ground state, we use the variational principle to find the equations for $\theta_{i}$ and $\phi_{i}: \delta H_{c} / \delta \theta_{i}=0$ and $\delta H_{c} / \delta \phi_{i}=0$. We see that $\phi_{i}=\phi_{j}$ and $\theta_{i}=\theta_{0}$ and/or $\theta_{0}+\pi$ at all the sites are solutions of the equations. Below we consider these solutions. In 1D it can be shown that these solutions give the lowest energy [13]. In $2 \mathrm{D}$ or $3 \mathrm{D}$ we speculate these solutions contain the lowest energy states, although a rigorous proof is absent. We set $\theta_{0}=0$ below and label all the lattice sites with $\theta_{i}=0$ by blacks, and all the sites with $\theta_{i}=\pi$ by whites. The bond Hamiltonian is then reduced to

$$
H_{c}(i j)= \begin{cases}J\left(\mathbf{T}_{i} \cdot \mathbf{T}_{j}+1 / 4\right), & \text { if } \theta_{i}=\theta_{j} \\ 0, & \text { if } \theta_{i}=\theta_{j} \pm \pi .\end{cases}
$$

Therefore, the coupled spin-orbital system is decomposed into disconnected black and white sites or blocks (collection of the connected same colored sites). In the same colored block, all the spins are parallel but the orbitals interact antiferromagnetically, and the interaction vanishes between different blocks. The total energy of the system is then the simple sum of these blocks. This greatly simplifies the calculations.

Let us first consider a two-site problem. The ground state of Eq. (5) is an orbital singlet with total spin $2 s_{t}$. We call this two-site state an OVB, whose energy is $-0.5 \mathrm{~J}$. There are two competing terms in Eq. (5). One is the spin-orbital coupled term, $\cos ^{2}\left(\Theta_{i j} / 2\right) \mathbf{T}_{i} \cdot \mathbf{T}_{j}$, which favors FM spins and antiferromagnetic (AFM) orbitals. The other is the spin interaction term $\cos ^{2}\left(\Theta_{i j} / 2\right) / 4$, which favors AFM spins. Because of this competition, the ground state of more than two sites is generally not a uniform FM spin state as we see explicitly below. In the 1D chain, we divide the chain into blacks and whites and calculate the lowest energies of these spin configurations. In particular, we consider the configurations with alternating black and white segments of $n$ sites (see Fig. 1a). The total energy of the chain is the sum of these independent segments. The ground state energies of these segments are calculated by using the exact numerical diagonalization method from $n=2$ to 16 . The results are plotted in Fig. 2. The energies of the even $n$ segments are lower than those of the odd $n$ ones. The ground state is an OVB solid with alternating spins up and down with an energy $-0.25 \mathrm{~J}$ per bond, much lower than that of the uniform FM spin state corresponding to $n \rightarrow \infty$. The energy of the latter state can be deduced from the result of the Bethe ansatz solution, and it is $(1-2 \ln 2) J / 2=-0.193 J$ per bond. Our numerical results approach the exact result rapidly as $n$ increases. Including the spin quantum fluctuation, we expect that the 1D AFM spin long range order be destroyed based on the dimensionality consideration and the calculation of the linearized spin wave theory. The system is then described by a spin- $2 s_{t}$ chain of the OVBs with AFM Heisenberg coupling $J /\left(32 s_{t}^{2}\right)$. Since $2 s_{t}$ is an integer, the ground state is a Haldane's gap state. The quantum ground state has twofold degeneracy corresponding to the translational invariance by one lattice constant in the OVBs.

In a 2D square lattice, we consider various spin configurations including an alternating colored two-site bond state (Fig. 1b), an alternating four-site plaquette state (Fig. 1c), stripes, and the uniform FM states. The ground states are found to be highly degenerate [14]. The lowest energy state in Fig. 1b is an OVB state, and the lowest energy state in Fig. 1c is a plaquette orbital singlet state whose orbital is given by [15] $\mathbf{t}(12) \mathbf{t}(34)-\mathbf{t}(14) \mathbf{t}(23)$, with $\mathbf{t}(12)$ representing an orbital singlet of sites 1 and 2 . The OVB, the plaquette orbital, and their mixed states are degenerate and have energy $-0.125 \mathrm{~J}$ per bond. The uniform FM spin state has a much higher energy $\left\langle\mathbf{T}_{i} \cdot \mathbf{T}_{j}+1 / 4\right\rangle_{2 \mathrm{D}}=-0.085 \mathrm{~J}$ per bond as estimated from the known result of the $2 \mathrm{D}$ spin- $1 / 2$ model [16]. The OVB state is also found to have

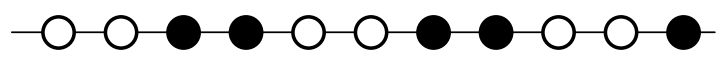

(a)

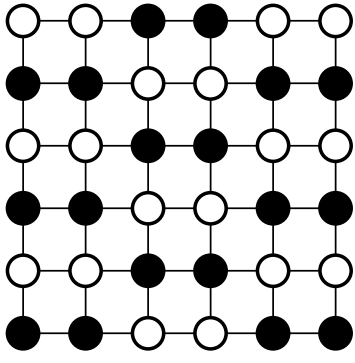

( b )

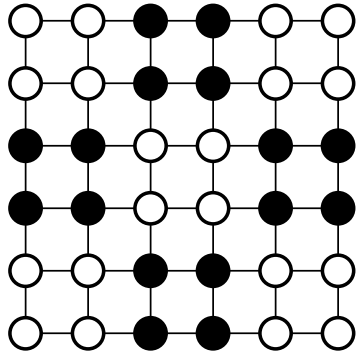

(c)
FIG. 1. Illustration of several possible lowest energy states for the model. Black sites represent spin up, and white sites represent spin down. (a) A one-dimensional OVB solid. (b) A two-dimensional OVB solid. (c) A two-dimensional plaquette OVB solid. 


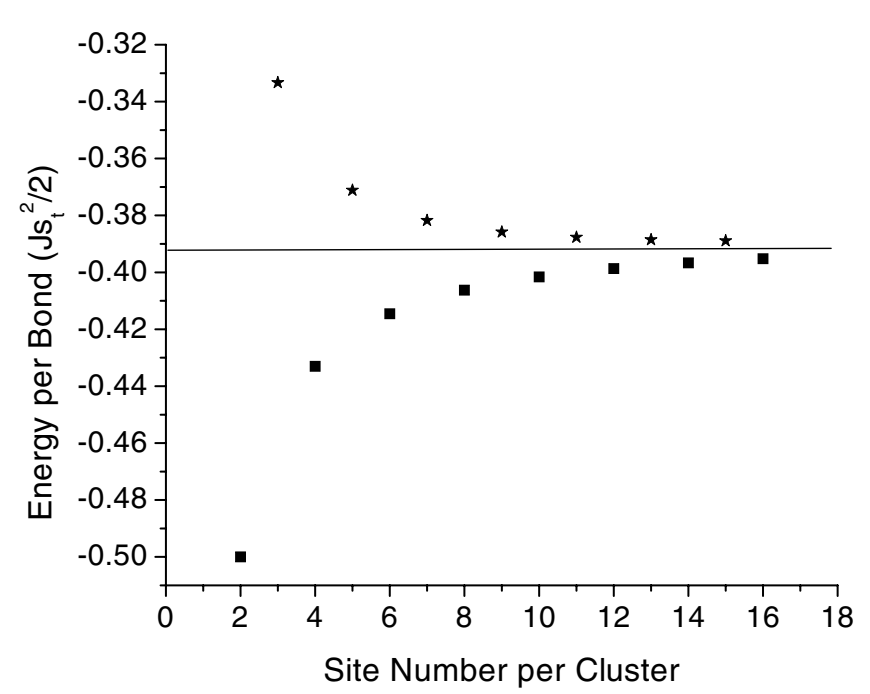

FIG. 2. Energy per bond $\left(J s_{t}^{2} / 2\right)$ versus the site number per cluster on a one-dimensional chain. The solid line corresponds to the exact energy of one-dimensional chain by means of Bethe ansatz.

the lowest energy in the 3D cubic lattice. The large degeneracy of the ground states found in the classical spin limit in the higher dimension is removed when the spin quantum fluctuation is included. We have used the spin wave theory to calculate the energy correction to the classical spin states in 2D square lattice and found it to be $-0.01157 \mathrm{~J} / \mathrm{s}_{t}$ for the OVB state and $-0.01012 \mathrm{~J} / s_{t}$ for the plaquette orbital state. Therefore the quantum spin fluctuation favors the OVB state. In $2 \mathrm{D}$ and $3 \mathrm{D}$, the AFM spin long range order is expected to survive from quantum fluctuation. We thus conclude that the ground state of Eq. (4) in 2D and $3 \mathrm{D}$ is the OVB state with AFM ordered spins between the nn bonds.

We now turn to discuss the possible relevance of our theory to some transition metal oxides. We first examine the ground state of cubic vanadate $\mathrm{LaVO}_{3}$. The observed magnetic order of the vanadate is of the $C$-type AFM phase (FM along the $c$ axis and AFM in the $a-b$ plane), arising from a practically undistorted structure above the Néel temperatures $[17,18]$. To explain the unusual magnetic ordering, Khaliullin et al. [9] recently proposed a spin-orbital Hamiltonian for the oxides. In the limit $\eta=J_{H} / U \rightarrow 0$ (the notations are the same as in their Letter) and along a given cubic axis, their model is reduced to Eq. (4) in the present Letter with $s_{t}=1$. The corresponding two equivalent orbitals for the coupling along the $c$ axis are $d_{z x}$ and $d_{y z}$, for example. Khaliullin et al. compared the ground state energies of the $C$-type and $G$-type (AFM in all three directions) AFM phases. They concluded that the $C$-type AFM is more stable at $\eta=0$ and is further stabilized at $\eta>0$. In that work, the comparison between the $C$-type AFM and the OVB state was not included. As we discussed earlier, the ground state is an OVB state at $\eta=0$ in the large spin limit. We believe the experimentally observed $C$-type AFM in that compound is stabilized by the Hund's coupling. Indeed we have compared the energies of $C$-type AFM and OVB states in their model [Eq. (1) of Ref. [9]] and found that the $C$-type AFM has lower energy for $\eta>\eta_{c}$ with $\eta_{c} \approx 0.06$ [19]. It will be interesting to examine the higher order spin fluctuation or to use numerical techniques to verify if the large spin limit applies to the spin-1 system.

In the second example, we consider the spin-orbital model for $\mathrm{LaMnO}_{3}$ assuming the lattice is not distorted. This type of model has been studied by many authors and the ground state is $A$-type AFM (AFM along the $c$ axis and FM within $a-b$ planes) [20]. Here we point out that the $A$-type ordering may be obtained by treating the Hund's rule coupling as a small perturbation. The effective Hamiltonian describing spin $S=2$ and the two degenerate $e_{g}$ orbitals of the Mn ion is given in the limit $J_{H} \rightarrow 0$ [5-8],

$$
H=\frac{J}{2 s_{t}^{2}} \sum_{\langle i j\rangle}\left(\mathbf{S}_{i} \cdot \mathbf{S}_{j}+s_{t}^{2}\right)\left(\tau_{i}^{\gamma}+\frac{1}{2}\right)\left(\tau_{j}^{\gamma}+\frac{1}{2}\right),
$$

where $\gamma$ is along $j-i$ and the orbital operators $\tau_{i}^{\gamma}=$ $\cos \left(2 m_{\gamma} \pi / 3\right) \mathbf{T}_{i}^{z}-\sin \left(2 m_{\gamma} \pi / 3\right) \mathbf{T}_{i}^{x}\left(m_{\gamma}=1,2,3\right)$ with eigenvalues $\pm \frac{1}{2}$. The ground state of this Hamiltonian in the large spin limit is highly degenerate. As the expectation value of $\left(\tau_{i}^{\gamma}+\frac{1}{2}\right)\left(\tau_{j}^{\gamma}+\frac{1}{2}\right)$ is always not less than zero, the low bound for the ground state energy is zero. Thus the $G$-, $C$-, and $A$-type AFM are all degenerate ground states with zero energy. When a small Hund's rule coupling is introduced, the high degeneracy in the ground state is removed. To simplify our problem, we keep the perturbation term due to the Hund's rule coupling up to the order $J J_{H} / U, \Delta H=-\left(J / 2 s_{t}^{2}\right) \sum\left(\mathbf{S}_{i} \cdot \mathbf{S}_{j}+s_{t}^{2}\right) h$, where $h$ is of the order $s J_{H} / U \ll 1$. Including $\Delta H$, the $A$-type AFM spin state is most favorable in energy. In that state, the orbitals are $C$-type antiferro-orbitally ordered: $d_{x^{2}-z^{2}} / d_{y^{2}-z^{2}}$ alternative in the $a-b$ plane and ferro-orbital along the $c$ axis. This state is favored because of the energy gain of $\Delta H$ from the bonds within the $a-b$ planes. Our calculation shows that the superexchange interaction including the Hund's coupling leads to a spin A-type and an orbital $C$-type structure. This state might be realized in $\mathrm{KCuF}_{3}$ [5]. Note that the orbital ordering pattern is different from that experimentally observed in $\mathrm{LaMnO}_{3}$ with a $d_{3 x^{2}-r^{2}} / d_{3 y^{2}-r^{2}}$ alternating ordering. The observed orbital ordering is due to strong Jahn-Teller distortion [21], but not due to the superexchange [1].

Finally, we examine an extended model of Eq. (4) in a cubic lattice to include the anisotropic coupling in a different axis and the Hund's rule coupling,

$$
H=\sum_{i, a} J_{\alpha}\left(\mathbf{S}_{i} \cdot \mathbf{S}_{i+a}+s_{t}^{2}\right)\left(\mathbf{T}_{i} \cdot \mathbf{T}_{j}+\frac{B}{4}\right),
$$

where $J_{\alpha}$ is the coupling strength along the direction $\alpha=$ $a, b, c$. At the symmetric point $B=1$ and $J_{a}=J_{b}=J_{c}$, 


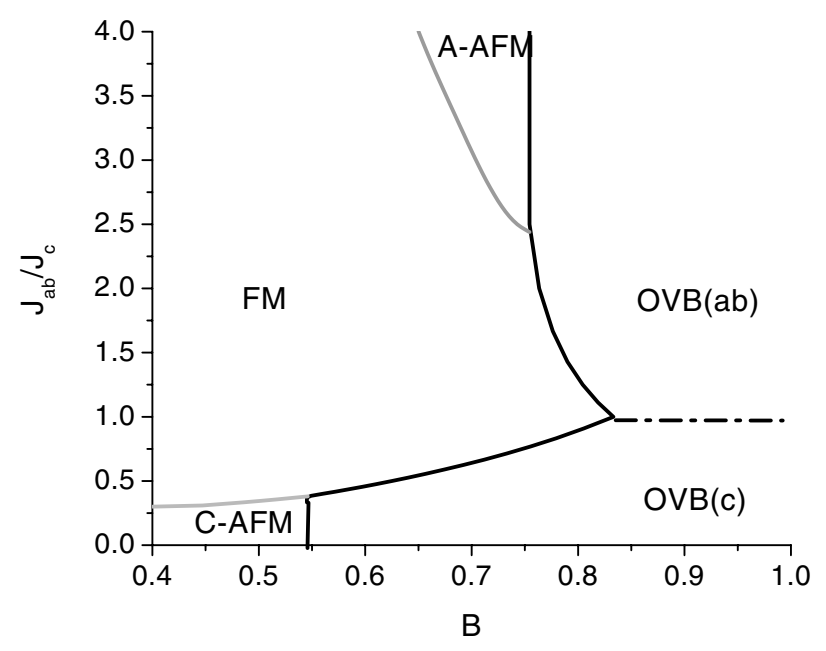

FIG. 3. The phase diagram for high spin systems with orbital degeneracy in Eq. (5). OVB $(a b)$ represents the OVBs aligning along the $a$ or $b$ axis, and $\mathrm{OVB}(c)$ represents the OVBs aligning along the $c$ axis.

the model is reduced to Eq. (4). In the presence of $J_{H}$, $B<1$ (see in $\Delta H$ in the previous example). We compare the ground state energies of various states including the OVB state, the uniform FM spin state, and the $A-, C-$, and $G$-type AFMs. In Fig. 3, we plot the phase diagram for the lowest energy states in the parameter space $B$ and $\eta=J_{a b} / J_{c}$. The OVB state is found to be a stable ground state in a finite parameter space around the symmetry point. It will be interesting to find an experimental realization of the OVB state. Since the OVB state breaks the translational symmetry, we expect the electron-lattice interaction may accompany a crystal structure phase transition to result in shorter and longer bonds. At a larger $\eta$ and smaller $B$, the $C$-type AFM spin state is realized, in which the orbitals are disordered liquid. At a smaller $\eta$ and smaller $B$, the $A$-type AFM becomes stable, in which the orbitals have a long range AFM order in the $x-y$ plane, and FM along the $z$ axis. The phase diagram is thus very rich, reminiscent of some of the features in the transition metal oxides.

In conclusion, we have examined the ground state of a coupled high-spin orbital model from the large spin limit point of view. A novel orbital valence bond state is found to be stable in a certain parameter region and may be realized in future experiments. The anisotropic model contains both $C$-type and $A$-type AFM spin states among others. Our method should be useful in the study of more realistic models when Hund's rule coupling is weak. The large spin approach emphasizes orbital quantum fluctuation over spins, and special care is needed to apply the method to the spin- $1 / 2$ case. In the $S U(4)$ limit, there is a permutation symmetry between spin and orbital, and we do not expect the method to work. The quantum fluctuation may drive the two degenerated spin and orbital valence bond states to a quantum state with more intricate correlations. In fact, from the Bethe ansatz solution, the 1D chain has a gapless SU(4) liquid ground state, which is not dimerized [22].

We thank Jian Wang and Michael Ma for useful discussions. This work was in part supported by RGC Grant No. HKU 7088/01P from Hong Kong, by U.S. DOE Grant No. DE/FG03-01ER45687, and by the Chinese Academy of Sciences.

[1] Y. Tokura and N. Nagaosa, Science 288, 462 (2000).

[2] E. Saitoh et al., Nature (London) 410, 180 (2001).

[3] Y. Q. Li et al., Phys. Rev. Lett. 81, 3527 (1998).

[4] C. Castellani et al., Phys. Rev. B 18, 4945 (1978).

[5] K. I. Kugel' and D. I. Khomskii, Fiz. Nizk. Temp. 6, 207 (1980) [Sov. J. Low Temp. Phys. 6, 99 (1980)].

[6] S. Ishihara et al., Physica (Amsterdam) 263C, 130 (1996).

[7] L. F. Feiner et al., Phys. Rev. Lett. 78, 2799 (1997).

[8] S. Q. Shen and Z. D. Wang, Phys. Rev. B 61, 9532 (2000).

[9] G. Khaliullin et al., Phys. Rev. Lett. 86, 3879 (2001).

[10] This method was implicitly used by some authors.

[11] T. M. Rice, Spectroscopy of Mott Insulators and Correlated Metals, edited by A. Fujimori and Y. Tokura (Springer, Berlin, 1995).

[12] K. Kubo and N. Ohata, J. Phys. Soc. Jpn. 33, 21 (1972); S. Q. Shen and Z. D. Wang, Phys. Rev. B 58, R8877 (1998).

[13] In 1D and for an arbitrary orbital configuration, we can assign a spin configuration in which two neighboring spins are parallel if $\left(\mathbf{T}_{i} \cdot \mathbf{T}_{j}+1 / 4\right)$ is negative, and are antiparallel otherwise. Such a spin configuration gives the lowest energy for each bond in the corresponding orbital state. Therefore, the solutions we consider contain the lowest energy states.

[14] We have evaluated the energies for a series of clusters consisting of up to 18 sites by exact diagonalization. Similar to the 1D chain, larger-size clusters tend to have higher energies than a collection of smaller-size clusters with the same number of lattice sites. For example, the energy for a $2 \times 4$ cluster is higher than the energy for two $2 \times 2$ or four $1 \times 2$ clusters.

[15] K. Ueda et al., Phys. Rev. Lett. 76, 1932 (1996).

[16] S. D. Liang, Phys. Rev. 42, 6555 (1990).

[17] A. V. Mahajan et al., Phys. Rev. B 46, 10966 (1992).

[18] H. C. Nguyen et al., Phys. Rev. B 52, 324 (1995).

[19] After we completed the work, we learned that the valence bond state was recently also considered by G. Khaliullin et al. in a paper in preparation (private communication).

[20] J. Rodriguez-Carvajal et al., Phys. Rev. B 57, R3189 (1998); also see Colossal Magnetoresistive Oxides, edited by Y. Tokura (Gordon and Breach, New York, 2000).

[21] P. B. Allen et al., Phys. Rev. B 60, 10747 (1999).

[22] B. Sutherland, Phys. Rev. B 12, 3795 (1975). 